Acknowledgment of Ad Hoc Reviewers 2021

\title{
Principal Ad Hoc Reviewers
}

The Editor and Associate Editors wish to acknowledge the following ad hoc reviewers. These individuals reviewed four or more manuscripts for the Journal of Occupational Health Psychology during the period between October 2020 and September 2021 and were designated Principal Ad Hoc Reviewers in recognition of their service.

Andrew Bennett

Jacquelyn M. Brady

Kimberly A. French

Daniela Andrei

Cristian Balducci

Peter A. Bamberger

Lisa Baranik

Daniel Beal

Myriam N. Bechtoldt

Vanessa Beck

Margaret Beier

Mindy Bergman

Jeremy M. Beus

Renzo Bianchi

Alyssa Birnbaum*

Petri Bockerman

Janet A. Boekhorst

Jonathan Booth

Rebecca Brauchli

Veerle Brenninkmeijer

Thomas W. Britt

Douglas Brown

Claudia Buengler

Vanessa Burke

Erica Carleton

Jack Carson

Tristan William Casey

Anne Casper

Carmina Castellano-Tejedor

Malissa Amy Clark

Stacey Conchie

Tori L. Crain

Jessica de Bloom

Nele De Cuyper

Maike Debus

Caitlin A. Demsky

Deanne Den Hartog

Jan Dettmers

Lindsay Dhanani
Lixin Jiang

Katharina Naswall

Shannon Taylor

\section{Ad Hoc Reviewers}

Stefan Diestel

Michael DiStaso*

Cecelia Dotzler*

Jordi Escartin

Kevin Eschleman

Yanran Fang*

Samuel Farley

Caterina Fiorilli

Paul E. Flaxman

Keaton A. Fletcher

Stephanie Gilbert

Nicolas Gillet

Gary W. Giumetti

Sharon Glazer

Steve Granger

Maja Graso

Matthew J. Grawitch

Lindsey Greco

James Grosch

Brian Gunia

Andrew Hafenbrack

Victor Y. Haines

Jonathon R. B. Halbesleben

Juliet Hassard

Lucy Headrick*

Raphael M. Herr

Rebecca Hewett

Kristin Hildenbrand

Lisle S. Hites

Camilla Holmvall

Kristin A. Horan

Guo-hua Huang

Sofya Isaakyan

Nina M. Junker

Jana Kühnel
Oliver Weigelt

Zhiqing E. Zhou
Seth Kaplan

Maria Karanika-Murray

Tal Katz-Navon

Anita C. Keller

Joel Koopman

Bettina Kubicek

Richard Landers

Jessica Lang

Jin Lee

Allan Lee

KiYoung Lee

Marcie LePine

Aleksandra Luksyte

Gil Luria

Denisa Luta

Zhanna Lyubykh

Shannon Marlow

Robin Martin

Sebastiano Massaro

Kelsey L. Merlo

Nathan Nguyen

Morten Birkeland

Nielsen

Kimberly O'Brien

Roman Pauli*

Stephanie C. Payne

Yisheng Peng

Sara Jansen Perry

Pascale Peters

Sabine Raeder

Jennifer Ragsdale

Usman Raja

Tara C. Reich

Gudrun Reindl

Dorota Reis 
Erin M. Richard

David A. Richards

Sonja Rispens

Wladislaw Rivkin

Lara Christina Roll

Cort W. Rudolph

Gordon Sayre

Gordon Schmidt

Annika Scholl

Birgit Schyns

Eva Selenko

Francesco Sguera

Rachel Williamson Smith

* Denotes a co-reviewer.
Andrew Spark

Christine Julia Syrek

Susanne Tafvelin

Sara Tement

Stefan T. Thau

Geoff Thomas

Thomas M. Tripp

Sergio A. Useche

Margot van der Doef

Danielle van Jaarsveld

Laura Venz

Pisitta Vongswasdi
Ivana Vranjes

Megan Walsh

Hai-Jiang Wang

Karyn L. Wang

Christopher W. Wiese

Brad Wipfli

Cindy Wu

Dana Yagil

Zhenyu Yuan

Dieter Zapf

Jingtao Zhu 